\title{
Proteus mirabilis sp. intestinal microflora grow in a dextran sulfate sodium-rich environment
}

\author{
YOSHIO ARAKI ${ }^{1}$, KENICHI MUKAISHO ${ }^{1}$, HIROYUKI SUGIHARA ${ }^{1}$, \\ YOSHIHIDE FUJIYAMA ${ }^{2}$ and TAKANORI HATTORI ${ }^{1}$ \\ Departments of ${ }^{1}$ Pathology and ${ }^{2}$ Internal Medicine, Shiga University \\ of Medical Science, Seta Tsukinowa, Otsu, Shiga 520-2192, Japan
}

Received July 7, 2009; Accepted August 19, 2009

DOI: 10.3892/ijmm_00000331

\begin{abstract}
The pathogenic mechanisms responsible for inflammatory bowel disease, especially ulcerative colitis (UC), are poorly understood. As an animal model, the oral administration of dextran sulfate sodium (DSS) induces colitis, which exhibits several clinical and histological features similar to UC. However, the pathogenic factors responsible for DSSinduced colitis and above all, the intestinal microflora in this colitis remain unclear. Therefore, we investigated the relationships between DSS and the intestinal microflora in this study. First, the depolymerization of DSS in mouse feces was analyzed using a pyridylamino-labeling (PA-DSS) and HPLC system. Next, a bacteriological study of the fecal contents using DSS-rich media and subsequently a classification using 16S rRNA were performed. Surprisingly, DSS was depolymerized in mouse feces under aerobic conditions, not under anaerobic conditions. Several kinds of microflora were suggested to be involved in this depolymerization. In particular, Proteus mirabilis can grow in DSS-rich media and has an ability to desulfonate and depolymerize DSS. Then, we produced chemically-modified $M r 2500$ DSS from native $M r 5000$ DSS. This depolymerized $M r 2500$ DSS was administered orally to mice and the colitis was evaluated histologically. The cytotoxicity of $M r 2500$ DSS on Caco-2 cells was also investigated. $M r 2500$ DSS induced weaker colitis in mice and weak cytotoxicity on Caco- 2 cells as compared to $\mathrm{Mr} 5000 \mathrm{DSS}$. These findings give insight into the mechanisms responsible for DSS-induced colitis, especially with respect to the molecular mass of DSS.
\end{abstract}

\section{Introduction}

Dextran sulfate sodium (DSS) has been used in animals to induce experimental colitis (1). The DSS-induced colitis model

Correspondence to: Dr Yoshio Araki, 6-13-20 Aoyama, Otsu, Shiga 520-2101, Japan

E-mail: yir0828@belle.shiga-med.ac.jp

Key words: dextran sulfate sodium-induced colitis, Proteus mirabilis, depolymerization, Caco-2 cell line, cytotoxicity, molecular mass is favored because of the high reproducibility of the colitis lesions and because these lesions are induced mainly in the left colon in a fashion similar to human ulcerative colitis (UC) (1). Therefore, DSS is widely used in investigations on the pathogenesis of UC and the efficacy of therapeutic agents. DSS is a heparin-like sulfated polysaccharide containing $\sim 17 \%$ sulfur, with up to three sulfates per glucose molecule (Fig. 1) (2). With respect to the pathogenic factors in this colitis model, previous studies have postulated the importance of various factors such as local immunological disturbances (3), the activation of mucosal macrophages (4), effects related to the strong negative-charge of DSS (5), obliteration of the crypt lumina (6) and changes in the intestinal microflora (7). Our previous studies also have postulated the importance of various factors such as the local involvement of reactive oxygen species $(8,9)$, short chain fatty acids (SCFA) (10) and cell cycle arrest (11). However, the precise etiology of this model remains unclear.

We previously reported that the microflora in the fecal contents was altered in DSS-induced colitis. In that study, Eubacterum, Bifidobacterium and total anaerobes were all significantly decreased, although Lactobacillus is significantly increased in DSS-induced colitis (12). In addition, even certain dietary fiber (germinated barley foodstuff) can alter the intestinal microflora, produce SCFA, subsequently colitis improved in human and DSS-induced colitis (13-16). Probiotics have been reported to have therapeutic effects for both diseases $(10,17)$. These results strongly suggest that the intestinal microflora play a certain role in intestinal inflammation.

First, it remains unknown whether some microflora can grow in a DSS-rich environment. If some microflora can grow in a DSS-rich environment, how do these microflora influence the luminal environment? This question seems very important to the pathogenic mechanisms of DSS-induced colitis. Therefore, we planned the present experiments in order to answer this question.

\section{Materials and methods}

Chemicals. DSS (Mr 5000, total sulfur 15.0-20.0\%), 2-aminopyridine, phthalic acid and tetra-n-buthylammonium hydroxide were obtained from Wako Pure Chemical (Osaka, Japan). Sodium cyanoborohydride were obtained from Nacalai Tesque 
Inc. (Kyoto, Japan). DSS (Mr 8000 or 10000) and D-glucose 3-sulfate was obtained from Sigma Chemical Co. (St. Louis, USA).

Animals. We used the fecal contents of specific pathogen-free male BALB/cA Jcl mouse, 6 weeks old, which were purchased from Nippon Clea Inc. (Tokyo, Japan). They were housed in a room with controlled temperature $\left(20-22^{\circ} \mathrm{C}\right)$, humidity (50-60\%) and a preset light-dark cycle (12 h: $12 \mathrm{~h})$. The experimental protocol was approved by the Animal Care and Use Committee of the Shiga University of Medical Science.

Labeling of DSS using 2-amynopyridine. The pyridylamination of the reducing termini of sugar chains has been useful for structural analysis and metabolic studies of $\mathrm{N}$ - or $\mathrm{O}$ glycosidically linked sugar chains (18). We used a labeling system of DSS in order to investigate the metabolism of DSS. Labeling of DSS using 2-amynopyridine was carried out according to our previous study (19). Briefly, $1.0 \mathrm{~g}$ of DSS was dissolved in $25 \mathrm{ml}$ of a 2-aminopyridine solution (prepared by mixing $5 \mathrm{~g}$ of 2-animopyridine, $3.8 \mathrm{ml}$ of conc. $\mathrm{HCl}$ and $11 \mathrm{ml}$ of distilled water, with a final $\mathrm{pH}$ of 6.7) and coupled with 2 -aminopyridine at $100^{\circ} \mathrm{C}$ for $13 \mathrm{~min}$. After the addition of $1.5 \mathrm{ml}$ of a reducing reagent (prepared by mixing $600 \mathrm{mg}$ of sodium cyanoborohydride and $1.5 \mathrm{ml}$ of distilled water just before use), the reaction mixture was heated at $90^{\circ} \mathrm{C}$ for $20 \mathrm{~h}$ for reductive amination. Four volumes of $\mathrm{NaCl}$-saturated ethanol were then added to the reaction mixture. The resulting precipitate was recovered by centrifugation at 3,000 rpm for $5 \mathrm{~min}$ and washed with ethanol 10 times to remove excess reagents. D-glucose 3 -sulfate was also labeled using 2-animo-pyridine as a monomer of sulfated polysaccharide.

HPLC conditions. An HPLC apparatus LC6A (Shimadzu, Kyoto, Japan) was used. The elution time of DSS was determined by gel filtration chromatography on a Cosmosil 5Diol-120 Packed column $(7.5 \times 300$ mm; Nacalai Tesque Inc). Since DSS is strongly negatively-charged polysaccharide in water, the interaction between DSS and column is suggested on this HPLC system. Therefore, 0.2 M phosphate buffer ( $\mathrm{pH}$ 3.0) was used as the mobile phase (19). The mobile phases were delivered isocratically at a flow rate of 1.0 $\mathrm{ml} / \mathrm{min}$. The column temperature was maintained at $60^{\circ} \mathrm{C}$ throughout this experiment. For the detection of pyridylamino-DSS (PA-DSS), an RF-535 fluorescence detector (Shimadzu) at excitation and emission wavelengths of 320 and $400 \mathrm{~nm}$, respectively, was used.

DSS depolymerization in the feces. Mouse fecal samples were immediately collected after defecation. A total of $2 \mathrm{ml}$ of distilled 5\% PA-DSS solution was added to $1 \mathrm{~g}$ of the sample. Under aerobic or anaerobic conditions, the diluted samples were incubated for $48 \mathrm{~h}$. After the incubation period, the samples were centrifuged at $10,000 \mathrm{rpm}$ for $10 \mathrm{~min}$. The supernatant was then filtered through a membrane filter (pore size $0.45 \mu \mathrm{m}$ ) and was injected into the HPLC columns. The PA-DSS in the samples was monitored using a fluorescence detector.

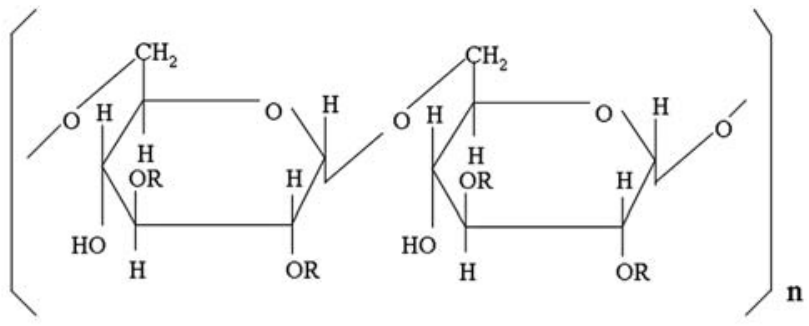

R: $\mathrm{SO}_{3} \mathrm{Na}$ or $\mathrm{H}$

Figure 1. Structure of dextran sulfate sodium (DSS).

Bacteriological study of the fecal contents. We produced a novel DSS-rich solid medium composed of 4\% DSS, $0.5 \%$ polypepton and $1.5 \%$ agar. The fecal contents were collected and then incubated in an aerobic or anaerobic environment. The anaerobic strains of microflora were investigated using a slight modification of previous methods $(20,21)$. Briefly, samples were serially diluted with a dilution solution under a $\mathrm{CO}_{2}$ environment. The plated microflora were then incubated in an anaerobic environment (AnaeroPack, Mitsubishi Gas Chemical Co., Tokyo, Japan) at $37^{\circ} \mathrm{C}$ for $72 \mathrm{~h}$. Differentiation of the microflora was performed by: i) an investigation of the colony morphology; ii) gram staining; iii) an investigation of the growth under aerobic conditions; and iv) an investigation of reactions in media consisting of p-nitrophenyl sulfate.

Classification using 16S rRNA. We obtained some microflora from DSS-rich solid media and then performed PCR to amplify a portion of the $16 \mathrm{~S}$ rRNA gene using specific primers. DNA was extracted from the microflora by using the SOIL for Beads Beating kit (Nippon Gene Co., Ltd., Tokyo, Japan). For amplification of the 16S rRNA, DNA was amplified using two primers: 10F: 5'-GTTTGATCCTGGC TCA-3', 800R: 5'-TACCAGGGTATCTAATCC-3'. The firstround amplification included 30 cycles of denaturation at $94^{\circ} \mathrm{C}$ for $30 \mathrm{sec}$, annealing at $72^{\circ} \mathrm{C}$ for $1 \mathrm{~min}$ and chain extension at $72^{\circ} \mathrm{C}$ for $3 \mathrm{~min}$. The amplification products were then analyzed by agarose gel electrophoresis. The RNA obtained was purified using a commercial kit and sequenced twice with an ABI 3100 genetic analyzer (Model 3100; Applied Biosystems, Foster City, CA, USA). The 16S rRNA sequences obtained were compared by BLAST (www.ncbi.nlm.nih.gov/BLAST/) with sequences available at GenBank. Calculation of the distance matrices and construction of a phylogenetic tree were performed with MEGA 3.1 software (www.megasoftware.net). A phylogenetic tree was constructed by the neighbor-joining method and the distance matrices for the aligned sequences were calculated by the Kimura 2-parameter method.

Capability of microflora obtained from DSS-rich solid media to depolymerize PA-DSS. We investigated the capability of microflora obtained from DSS-rich solid media to depolymerize PA-DSS. The microflora were incubated in a PADSS-rich liquid medium composed of 5\% PA-DSS, $0.5 \%$ polypepton and $0.05 \%$ sodium dihydrogenphosphate for $48 \mathrm{~h}$ under aerobic conditions. After incubation, the supernatant was analyzed by HPLC. 
Chemically depolymerized Mr $2500 \mathrm{DSS}$. We previously reported a method to depolymerize chemically $\mathrm{Mr} 5000 \mathrm{DSS}$ (22). $M r 5000$ DSS was depolymerized to a $M r 2500$ moiety under $1 \mathrm{~N}$ acidic conditions. In addition, a small amount of sulfate was depleted from DSS under these conditions $(<10 \%)$.

Mouse Mr 2500 or 5000 DSS-induced colitis. The mice were allowed standard rodent food (CE-2, Nippon Clea Inc.) and drinking water ad libitum. At the beginning of the experiment, the mice were fed the standard diets (MF, Oriental Yeast Co,. Lt, Tokyo, Japan) containing 5\% (w/w of diet) $M r 25000$ or 5000 DSS for 8 days to compare the colonic damage $(n=5)$. During the experimental period, food intake and body weight were measured every other day. On the final day of the experiment, the rats were anesthetized with an intraperitoneal injection of pentobarbital sodium $(40 \mathrm{mg} / \mathrm{kg})$ and were sacrificed by cervical dislocation. After a laparotomy was performed, the large intestine was resected, cut along the anti-mesenteric border and irrigated with chilled saline. A specimen $(5 \times 5 \mathrm{~mm})$ at $2 \mathrm{~cm}$ distance from the anal margin (middle colon) was removed, frozen and cut into $5 \mu \mathrm{m}$ sections. Finally, the sections was stained with hematoxylin and eosin (HE) and observed under a microscope. The mucosal damage was determined according to a previously described method (23). Briefly, the following three parameters were used: surface epithelium loss, crypt destruction and inflammatory cell infiltration to the mucosa. A score of 0-4 was assigned to each of three parameters according to the extent and severity of change: $0=$ no change; $1=$ localized and mild; 2 = localized and moderate; 3 = extensive and moderate; $4=$ extensive and severe. The sum of the scores of the three parameters represented the mucosal damage score in each animal.

Cell culture. Caco-2 (a human colon cancer cell line) cells were purchased from the American Type Culture Collection (Rockville, USA). Passages 15-21 were used in this experiment. The Caco-2 cells were cultured in Dulbecco's modified Eagle's minimum essential medium (DMEM, pH 7.4) supplemented with $25 \mathrm{mM}$ glucose, $10 \%$ inactivated fetal bovine serum (FBS), $1 \%$ penicillin-streptomycin and $1 \%$ non-essential amino acid solution. The cells were maintained at $37^{\circ} \mathrm{C}$ in a humidified $5 \% \mathrm{CO}_{2}$ atmosphere.

Cytotoxicity (viability) tests. Cell viability assays were performed according to our previous method (11). Briefly, cells were inoculated in 96-multiwell plates (Costar, Corning, NY, USA) at a cell density of $1.2 \times 10^{5}$ cells/well. After cells reached confluence, cells were incubated with serially diluted and filter-sterilized $\mathrm{Mr} 2500$ or 5000 DSS in a growth medium for pre-determined time periods. Viability was assayed by a commercially available kit (Cell Titer 96 ${ }^{\mathrm{TM}}$ AQueous, Promega, Madison, USA), which depends on the physiologic reduction of MTS to formazan. Analyses were performed in triplicate. The most important thing here is that the $M r 5000$ PA-DSS is easily depolymerized to $M r 1800$ and $~ 70 \%$ of the sulfate groups are depleted from one DSS molecule following autoclave treatment (at $115^{\circ} \mathrm{C}$ for $15 \mathrm{~min}, 1.7 \mathrm{~atm}$ ). Therefore, autoclave treatment should be avoid for sterilization and filter treatment is recommended (22).
Statistical analysis. The results are presented as means \pm SEM. The variance was analyzed by the F test. Subsequently, Student's t-test for unpaired values was performed to compare the means of normally distributed data. The Mann-Whitney $\mathrm{U}$ test was also performed to compare the means of nonparametric or abnormally distributed data. Differences were regarded as statistically significant at $\mathrm{P}$-values $<0.05$.

\section{Results}

DSS depolymerization in the feces. Under aerobic conditions, native $M r 5000$ PA-DSS in the feces was depolymerized to a certain degree and two new peaks appeared in the chromatogram (Fig. 2A). The molecular masses of these peaks were speculated to be $\sim M r 1200$ and 750, respectively, according to the relationship between the elution time and the molecular mass of each PA-DSS and PA-D-glucose 3-sulfate moiety. In addition, the $M r 1200$ and 750 DSS were calculated to comprise $\sim 3.4$ and $6.0 \%$ of the depolymerized products from the total PA-DSS amount, respectively. Therefore, a total of $9.4 \%$ of the PA-DSS was depolymerized under these conditions. In contrast, under anaerobic conditions, this depolymerization was far weaker (Fig. 2B).

Bacteriological study of the fecal contents. Some microflora grew on the DSS-rich media. These microflora were gram negative. On the other hand, obvious microflora were not recognized in the anaerobic environment. These microflora obtained in the aerobic environment formed clear films on the growth media (or 'swarming motility') in GAM media, which indicated that these microflora were of Proteus sp. In addition, these flora had desulfonating activities toward p-nitrophenyl sulfate, which indicated that these microflora had arylsulfatase activity.

Classification using $16 S$ rRNA. The sequences obtained were similar to those of Proteus mirabilis. Fig. 3 shows a phylogenetic tree.

Capability of Proteus mirabilis to degrade PA-DSS. Fig. 2C shows a chromatogram of the supernatant from PA-DSS-rich liquid media, which showed a small peak after a PA-DSS peak. This result indicated that Proteus mirabilis had some capability to depolymerize PA-DSS.

Mouse Mr 2500 or 5000 DSS-induced colitis. After an oral administration of $M r 5000$ DSS, diarrhea occurred on days 3-4. Macroscopic examination of the colon revealed hyperemia, erosion and occasional tiny blood clots in the distal colon. Using HE staining, there was obvious evidence of inflammatory cell infiltration into the mucosa and submucosa in the distal portions on day 8 after the DSS administration (Fig. 4C). Entire crypt loss, surface epithelial loss and mucosal edema were also evident as compared to the control mice (Fig. 4A). On the other hand, after an oral administration of Mr $2500 \mathrm{DSS}$, the appearance of diarrhea was delayed until days 6-7. Macroscopic and microscopic examinations of the colon revealed that colitis in mice fed $M r 2500$ DSS was milder than in mice fed $M r 5000$ DSS (Fig. 4B). The mucosal damage quantified by a scoring system is shown in Fig. 4D. 
A
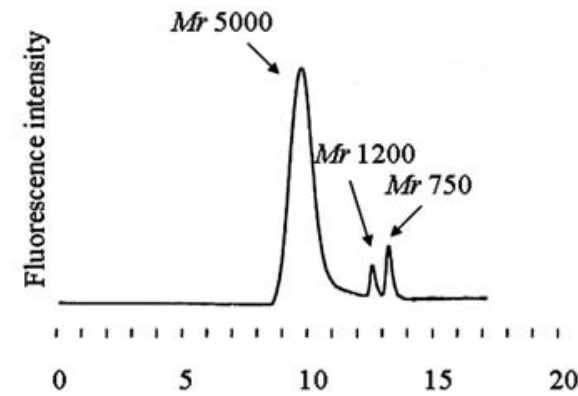

B

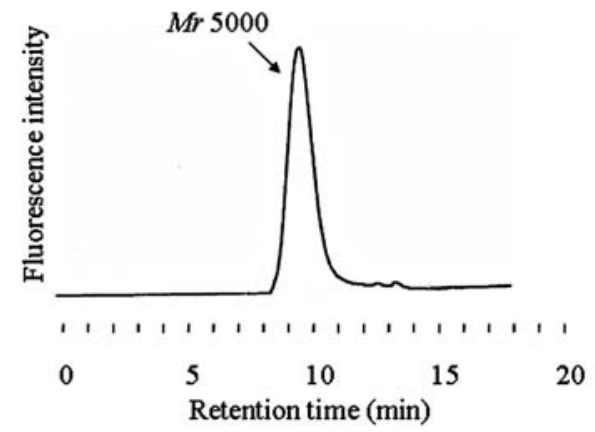

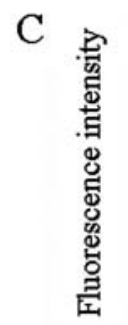
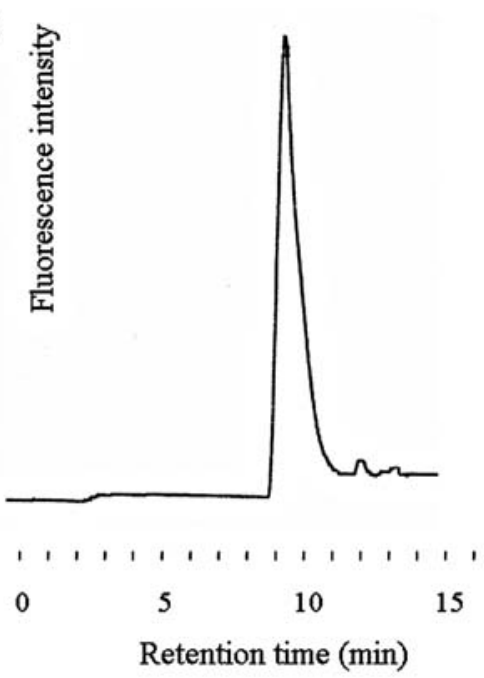

Figure 2. Gel filtration chromatograms of pyridylamino-DSS (PA-DSS). (A) Depolymerization of PA-DSS by mouse feces under aerobic conditions. (B) Depolymerization of PA-DSS by mouse feces under anaerobic conditions. (C) A chromatogram of supernatant obtained from PA-DSS-rich liquid media. PA-DSS was depolymerized by microflora. The HPLC conditions are described in Materials and methods.

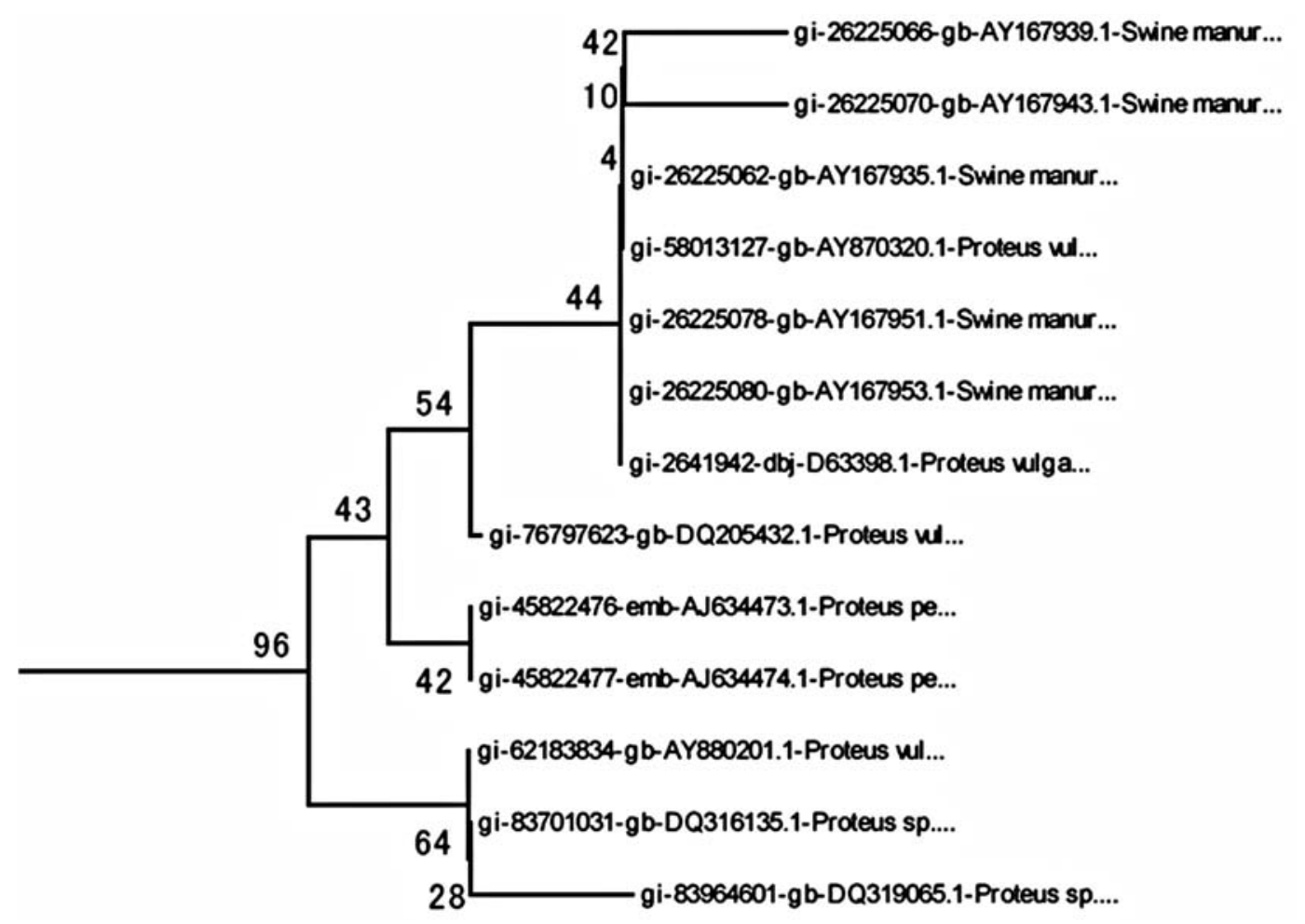

Figure 3. A maximum likelihood phylogenetic tree of the microflora, which grew on DSS-rich solid media. Analytical procedures are described in Materials and methods.

The difference between $\mathrm{Mr} 5000$ and 2500 was statistically significant. These results indicated that $M r 2500$ DSS induced far milder colitis than $M r 5000$ DSS.

Cytotoxicity (viability) tests. Fig. 5 shows Caco-2 survival curves for cells exposed to $M r 2500$ or 5000 DSS. The percent survival was expressed as a percentage of the growth compared to the corresponding untreated control cells at various incubation periods. The survival curves of cells exposed to Mr 5000 DSS at $0.1 \%$ were comparable to the controls, but cytotoxic effects became clear at 1-5\%. With respect to $\mathrm{Mr} 2500$ DSS, the cytotoxic effects were not detectable even at $5 \%$. 

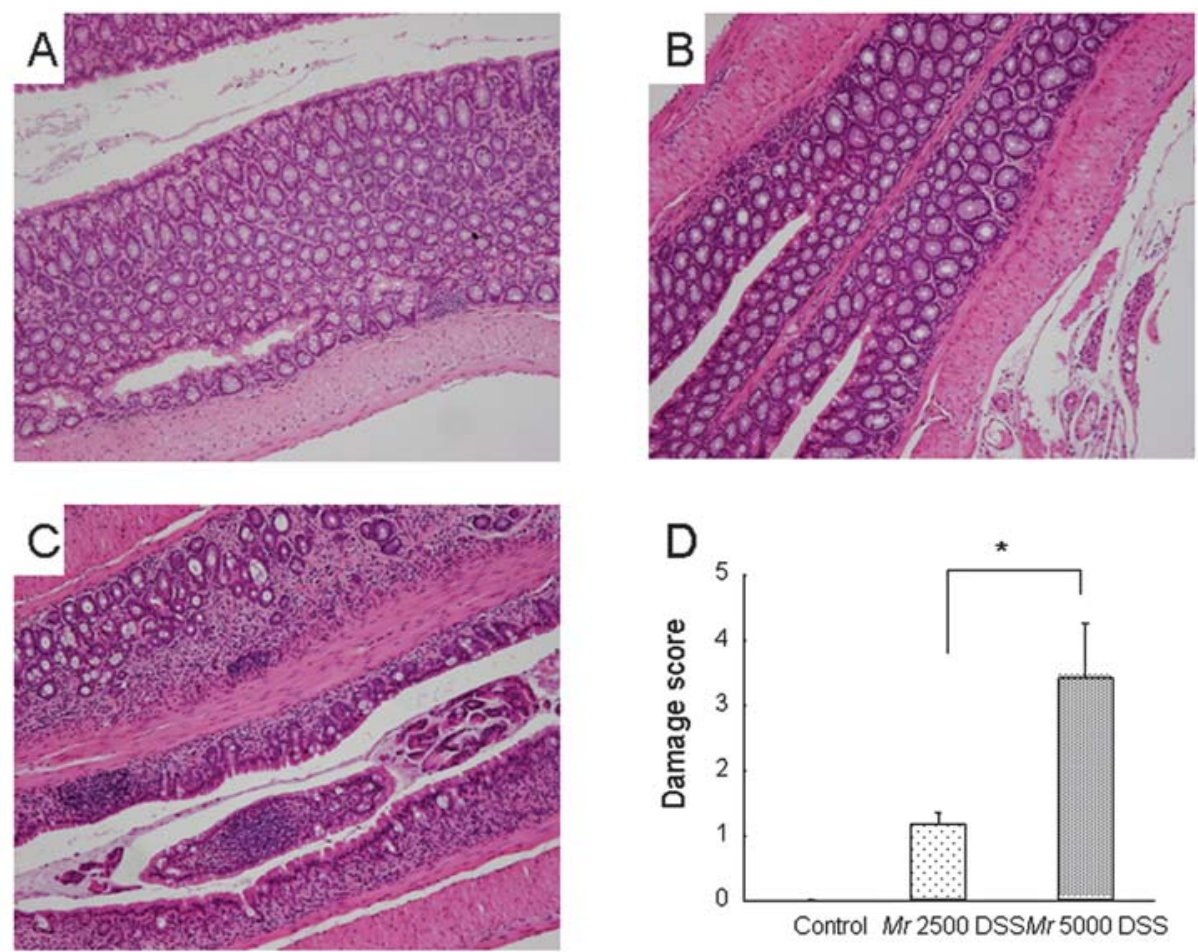

Figure 4. Mr 2500 or 5000 DSS-induced colitis. (A) HE staining of the colon in a control mouse. (B) HE staining of the colon in a $M r 2500$ DSS-induced colitis mouse. (C) HE staining of the colon in a Mr 5000 DSS-induced colitis mouse. (A-C) Magnification x100. (D) The mucosal damage quantified by a scoring system according to (23). All values are expressed as means \pm SEM. ${ }^{*} \mathrm{P}<0.05$.
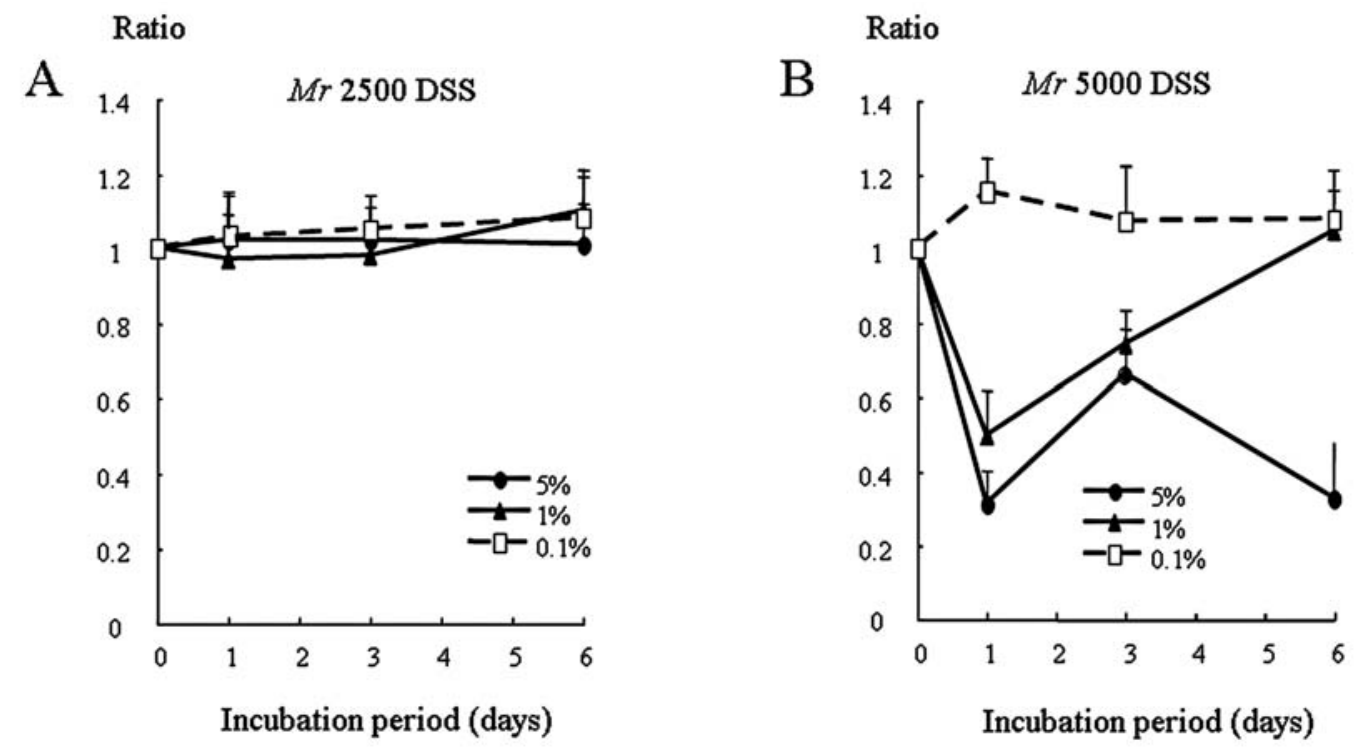

Figure 5. Cytotoxicity (viability) tests of $\mathrm{Mr} 2500$ or 5000 DSS on Caco-2 cells. The relative numbers of viable cells were determined using an MTS assay. The percent survival was expressed as a percentage of the growth compared to the corresponding untreated control cells at various incubation periods. Each point represents the mean of three determinations. (A) Survival curves of cells exposed to $M r 2500$ DSS at each DSS concentration. (B) Survival curves of cells exposed to $\mathrm{Mr} 5000 \mathrm{DSS}$ at each DSS concentration. All values are expressed as means \pm SEM. ${ }^{*} \mathrm{P}<0.05$.

Therefore, the cytotoxicity of depolymerized $\mathrm{Mr} 2500 \mathrm{DSS}$ on Caco-2 cells was very weak.

\section{Discussion}

The intestinal microflora have been suggested to play important roles in intestinal inflammation (24). In particular, the microflora in the fecal contents were altered in DSS- induced colitis (12). The precise meaning of these alterations, however, remains unknown. In other words, are these alterations a causative factor or just an indirect result of the colitis?

In this study, we discovered the following facts for the first time. i) Some microflora present in the feces can depolymerized DSS, especially under aerobic conditions. ii) Proteus mirabilis can grow in a DSS-rich environment and 
may be one of the microflora responsible for the depolymerized DSS in the feces. Moreover, Proteus mirabilis had arylsulfatase activity and could desulfonate DSS.

In general, Proteus mirabilis is a gram negative and facultatively anaerobic bacterium. It shows swarming motility and urease activity. Proteus mirabilis belongs to the Enterobacteriales, Gamma Proteobacteria. Proteus mirabilis is known to form crystallites in urine. Moreover, it has been reported that 1 out of 4 people have Proteus mirabilis in their gut lumen (25). However, it has not been reported that Proteus mirabilis can cause colitis.

Also in this study, iii) depolymerized $M r 2500$ DSS could not induce strong colitis any more. In addition, it was evident that $M r 2500$ DSS is less toxic on Caco-2 cells than Mr 5000 DSS in the cytotoxicity test.

We previously reported that $M r 50$ k DSS exhibited stronger cytotoxicity than $M r 5000$ DSS (11). Kitajima et al also reported in detail the relationship between the molecular mass of DSS and the features of colitis in mice. According to their study, the degree of colitis was stronger in the order of $M r 40$ k DSS > Mr 5000 DSS. $M r 500$ k DSS could not induce colitis any more (26). These results suggest that a certain range of molecular mass ( $\mathrm{Mr} 5000-50 \mathrm{k}$ ) may be required to induce colitis in vivo.

Taken together, it is strongly suggested that there are some kinds of microflora, for example Proteus mirabilis influencing the molecular mass of $M r 5000$ DSS in the feces. The depolymerized $M r 2500$ DSS can not induce strong colitis any more. Therefore, it is possible that Proteus mirabilis depolymerizes DSS and detoxifies DSS. In addition, the biological materials derived from Proteus mirabilis may be useful therapeutic agents for DSS-induced colitis.

In conclusion, the molecular mass of DSS is a very important factor in the induction and progression of DSSinduced colitis. Certain types of microflora including Proteus mirabilis sp. are suggested to influence the distribution of DSS molecular mass and progression of DSS-induced colitis.

\section{Acknowledgements}

We thank Mr. Noboru Urushiyama of Central Research Laboratory, Shiga University of Medical Science for his assistance with the HPLC analyses.

\section{References}

1. Elson CO, Sartor RB, Tennyson GS and Riddell RH: Experimental models of inflammatory bowel disease. Gastroenterology 109: 1344-1367, 1995.

2. Ricketts CR: Dextran sulphate - a synthetic analogue of heparin. Biochem J 51: 129-133, 1952.

3. Kobayashi K, Asakura H, Hamada Y, Hibi T, Watanabe M, Yoshida T, Watanabe N, Miura S, Aiso S and Tsuchiya M: T lymphocyte subpopulations and immunoglobulin-containing cells in the colonic mucosa of ulcerative colitis; a morphometric and immunohistochemical study. J Clin Lab Immunol 25: 63-68, 1988.

4. Abraham R, Fabian RJ, Golberg L and Coulston F: Role of lysosomes in carrageenan-induced cecal ulceratation. Gastroenterology 67: 1169-1181, 1976.

5. Watt J and Marcus R: Experimental ulcerative disease of the colon. Methods Achiev Exp Pathol 7: 56-71, 1975.

6. Iwanaga T, Hoshi O, Han H and Fujita T: Morphological analysis of acute ulcerative colitis experimentally induced by dextran sulfate sodium in the guinea pig: some possible mechanisms of cecal ulceration. J Gastroenterol 29: 430-438, 1994.
7. Onderdonk AB, Hermos JA and Bartlett JG: The role of the intestinal microflora in experimental colitis. Am J Clin Nutr 30: 1819-1825, 1977.

8. Araki Y, Andoh A and Fujiyama Y: The free radical scavenger edaravone suppresses experimental dextran sulfate sodiuminduced colitis in rats. Int J Mol Med 12: 125-129, 2003.

9. Araki Y, Sugihara H and Hattori T: The free radical scavengers edaravone and tempol suppress experimental dextran sulfate sodium-induced colitis in mice. Int J Mol Med 17: 331-334, 2006

10. Araki Y, Andoh A, Takizawa J, Takizawa W and Fujiyama Y: Clostridium butyricum, a probiotic derivative, suppresses dextran sulfate sodium-induced experimental colitis in rats. Int J Mol Med 13: 577-580, 2004.

11. Araki $\mathrm{Y}$, Sugihara $\mathrm{H}$ and Hattori T: In vitro effects of dextran sulfate sodium on a Caco-2 cell line and plausible mechanisms for dextran sulfate sodium-induced colitis. Oncol Rep 16: 1357-1362, 2006

12. Araki Y, Andoh A, Tsujikawa T, Fujiyama Y and Bamba T: Alterations in intestinal microflora, faecal bile acids and short chain fatty acids in dextran sulphate sodium-induced experimental acute colitis in rats. Eur J Gastroenterol Hepatol 13: 107-112, 2001.

13. Araki Y, Andoh A, Koyama S, Fujiyama Y, Kanauchi O and Bamba T: Effects of germinated barley foodstuff on microflora and short chain fatty acid production in dextran sulfate sodiuminduced colitis in rats. Biosci Biotechnol Biochem 64: 1794-1800, 2000.

14. Araki Y, Fujiyama Y, Andoh A, Koyama S, Kanauchi O and Bamba T: The dietary combination of germinated barley foodstuff plus Clostridium butyricum suppresses the dextran sulfate sodium-induced experimental colitis in rats. Scand J Gastroenterol 35: 1060-1067, 2000.

15. Araki Y, Andoh A, Fujiyama Y, Kanauchi O, Takenaka K, Higuchi A and Bamba T: Germinated barley foodstuff exhibits different adsorption properties for hydrophilic versus hydrophobic bile acids. Digestion 64: 248-254, 2001 .

16. Kanauchi O, Mitsuyama K, Homma T, Takahama K, Fujiyama Y, Andoh A, Araki Y, Suga T, Hibi T, Naganuma M, Asakura H, Nakano H, Shimoyama T, Hida N, Haruma K, Koga H, Sata M, Tomiyasu N, Toyonaga A, Fukuda M, Kojima A and Bamba T: Treatment of ulcerative colitis patients by long-term administration of germinated barley foodstuff: multi-center open trial. Int J Mol Med 12: 701-704, 2003.

17. Foligne B, Dessein R, Marceau M, Poiret S, Chamaillard M, Pot B, Simonet M and Daniel C: Prevention and treatment of colitis with Lactococcus lactis secreting the immunomodulatory Yersinia LcrV protein. Gastroenterology 133: 862-874, 2007.

18. Kon A, Takagaki K, Kawasaki H, Nakamura T and Endo M: Application of 2-aminopyridine fluorescence labeling to glycosaminoglycans. J Biochem 110: 132-135, 1991.

19. Araki Y, Andoh A, Fujiyama Y, Hata K, Makino J, Okuno T, Nakanura F and Bamba T: Application of 2-aminopyridine fluorescence labeling in the analysis of in vivo and in vitro metabolism of dextran sulfate sodium by size-exclusion highperformance liquid chromatography. J Chromatogr B Biomed Sci Appl 753: 209-215, 2001.

20. Campbell JM, Fahey GC Jr and Wolf BW: Selected indigestible origosaccharides affect large bowel mass, cecal and fecal shortchain fatty acids, $\mathrm{pH}$ and microflora in rats. J Nutr 127: 130-136, 1997.

21. Mitsuoka T: A Color Atlas of Anaerobic Bacteria (in Japanese). Soubunsha, Tokyo, pp53-119, 1984.

22. Araki Y, Mukaisyo K, Sugihara H and Hattori T: Decomposition of dextran sulfate sodium under alkaline, acidic, high temperature and high pressure conditions. Oncol Rep 20: 147-149, 2008.

23. Oda T: Role of mast cells in dextran sulfate sodium-induced experimental colitis in rats. J Kyoto Pref Univ Med 104: 1069-1082, 1995.

24. Hans W, Scholmerich J, Gross V and Falk W: The role of the resident intestinal flora in acute and chronic dextran sulfate sodium induced colitis in mice. Eur J Gastroenterol Hepatol 12: 267-273, 2000.

25. Adler JL, Burke JP, Martin DF and Finland M: Proteus infection in a general hospital. II. Some clinical and epidemiological characteristics with an analysis of 71 cases of Proteus bacteremia. Ann Intern Med 75: 531-536, 1971.

26. Kitajima S, Takuma S and Morimoto M: Histological analysis of murine colitis induced by dextran sulfate sodium of different molecular weights. Exp Anim 49: 9-15, 2000. 